\title{
Succeeding on the Canadian NCLEX-RN: Incorporating experiential learning approaches in the education of internationally educated nurses
}

\author{
Eva Hava Peisachovich * \\ School of Nursing, York University, Toronto, Ontario, Canada
}

\author{
Received: November 16, 2015 \\ Accepted: February 2, 2016 \\ Online Published: March 10, 2016 \\ DOI: $10.5430 /$ jnep.v6n7p101 \\ URL: http://dx.doi.org/10.5430/jnep.v6n7p101
}

\begin{abstract}
This study investigated the use of experiential education approaches in preparing internationally educated nurses (IENs) for the new registration examination in Canada, the National Council Licensure Examination for Registered Nurses (NCLEX-RN). The research comprised a purposive sample of ten participants in their first term of the IEN program at a local university in Ontario. The participants (a) wrote a pre and post NCLEX-RN style mock exam and (b) participated in a total of six clinical case scenarios, each followed by a brief questionnaire. The nursing-care case scenarios focused on six different systems (respiratory, cardiovascular, peripheral vascular, abdominal, neurologic, and endocrine); each scenario was followed by ten questions that were answered using an audience response system, namely clickers. A paired $t$ test was conducted to evaluate the impact of the application of both clinical-case scenarios and clickers on participants' scores; results demonstrated challenges associated with the transition to the American-based NCLEX-RN as the new national exam in Canada.
\end{abstract}

Key Words: NCLEX-RN, Canadian nursing registration, Canadian nurse licensing, Clickers, Internationally educated nurses

\section{INTRODUCTION}

The Canadian Council of Registered Nurse Regulators (CCRNR) recently decided to adopt the American-based National Council Licensure Examination for Registered Nurses (NCLEX-RN) as the new nursing registration exam for Canada (except Quebec). In January 2015 $5^{[1,2]}$ the NCLEX$\mathrm{RN}$, a computerized adaptive test, replaced the Canadian Registered Nurse examination (CRNE) as the national registration examination in Canada, giving rise to many challenges for both nursing students and faculty. The adoption of the new registration exam is of concern for nursing programs in Canada as the exam (a) is US based and, therefore, contains new test content and (b) utilizes a computer adaptive test delivery method, which requires increased application of
NCLEX-RN test plan questions in order to adequately prepare Canadian students to successfully pass the exam. This paper focuses on the latter challenge.

To date, tests and exams administered in Canadian nursing programs have largely aligned with the style of the CRNE, which employs a pen-and-paper format and features mostly multiple-choice questions with one correct answer. The computerized adaptive test format of the NCLEX-RN, then, represents a marked change. It is, therefore, imperative for both students and faculty that the structure of the new registration exam is embedded in the curriculum in order to successfully prepare students for it.

The purpose of the study under discussion was to investigate

*Correspondence: Eva Hava Peisachovich; Email: peva@yorku.ca; Address: School of Nursing, York University, Toronto, Ontario, Canada. 
whether experiential education approaches could enhance internationally educated nurses' (IENs) performance on the NCLEX-RN. A pretest-posttest research design was used to compare participants' performance on an NCLEX-RN style mock exam, administered both before and after an experiential education intervention. This intervention consisted of six different clinical scenarios, each proceeded by questions aligned with the current NCLEX-RN test plan or exam blueprint. The study is significant in today's nursing curriculum, as it provides insight regarding the actual challenges faced by nursing students, and particularly by IENs, as a result of this change to their registration and eligibility to practice in the province of Ontario and in the rest of Canada (except Quebec).

\subsection{Internationally educated nurses}

IENs often encounter barriers in meeting entry-to-practice requirements and in obtaining full licensure to practice. According to NCLEX-RN statistics, the first-time pass rates for international nurses have been much lower than for their US-educated counterparts. In 2015, the first-time pass rates for IENs and US candidates were $30.91 \%$ vs. $89.79 \%$, respectively. Similar differences were reported in earlier years; first-time pass rates for IENs and US-educated candidates were, respectively, $30.61 \%$ vs. $85.19 \%$ in 2013 and $28.88 \%$ vs. $84.93 \%$ in 2014. ${ }^{[3-5]}$ McGuire and Murphy, ${ }^{[6]}$ posit that language factors and lack of test-taking skills may contribute to IENs' low first-time pass rates.

In Ontario, nursing is the third-largest body with internationally trained members, ${ }^{[7]}$ yet IENs face challenges in obtaining the credential recognition required to practice in Canada. These challenges have been exacerbated by recent changes in CNO regulations and federal immigration, which have combined to reduce and potentially close off a number of immigration channels used by IENs to access the profession. One of these new regulations, the IEN Competency Assessment Program (IENCAP), was introduced in 2013..$^{[7,8]}$ The purpose of the IENCAP is to administer a standardized examination to test the judgment, skills, and knowledge of IENs seeking to be registered in the province of Ontario. ${ }^{[9]}$ The IENCAP includes both an Objective Structured Clinical Examination (OSCE) - a series of simulations to test entry-to-practice competencies-and a multiple-choice clinical examination designed to ensure that candidates' skills meet the national competencies set for entry-level registered nurses; $;{ }^{[9,10]}$ applicants who do not pass both the OSCE and the pen-and-paper exam must complete additional universitybased courses to fill competency gaps before they are eligible to write the licensing exam to practice in Ontario. Further complicating matters, the IENCAP provides candidates with 102 only one opportunity to be tested, yet there are limited spaces and programs available to prepare IENs for these exams. These recent changes, in concert with IENs' low first-pass rates on the NCLEX-RN, present a potential crisis for nurse educators in Canada.

Thus, it is essential that we create support and resources within the curriculum in order to strengthen both knowledge and application of content. These measures may translate into success on the OSCE and the NCLEX-RN-a possibility that emphasizes the significance of investigating how to better prepare candidates for this exam.

Recent research indicates that the application of experiential education in nursing curriculum is a useful and effective approach to providing students with real-life experiences within the context of teaching learning. ${ }^{[11,12]}$ In this study, the experiential-education approach comprised clinical-case scenarios proceeded by questions administered using clickers. Application of this approach was implemented to identify influence on pre and post scores on a mock NCLEX-RN.

\subsection{Overview and framework of NCLEX-RN}

The Detailed NCLEX-RN Test Plan for candidates outlines the content covered on the exam. According to the test plan, four processes are integrated through the test: nursing process, caring, communication and documentation, and teaching learning. Further, the framework of the NCLEX-RN is based on four major "client needs" categories (two of which are further divided into subcategories) to provide a universal structure for defining nursing actions and competencies that focus on clients in all nursing-care settings. ${ }^{[13]}$ The client-needs categories and subcategories are used to organize test content and categories. Table 1 provides the content categories, subcategories, and distribution percentage.

\subsection{Computer adaptive testing}

The NCLEX-RN is administered to candidates via computerized adaptive testing (CAT), a method of delivering examinations that uses computer technology and measurement theory. With CAT, each candidate's examination is "unique" as the adaptive computer technology selects items based on each candidate's level of comprehension, application, and analysis of items. ${ }^{[4]}$ The items are stored in a large item pool and are classified by test-plan category and level of difficulty. ${ }^{[4]}$ When a candidate answers an item, the computer interprets and estimates a candidate's ability, based on his or her previous answers, and selects the next item based on the candidate's estimated ability in the appropriate test-plan category. Each examination adapts to the candidate's knowledge and skills in fulfilling NCLEX-RN Test Plan requirements. ${ }^{[14]}$ 
Table 1. Distribution of Content of the 2013 NCLEXRN Test Plan (Adapted from the 2013 Detailed Test Plan for the National Council Licensure Examination for Registered Nurses)

\begin{tabular}{ll}
\hline NCLEXRN Client Needs Category & $\begin{array}{l}\text { Content Distribution } \\
\text { Percentage }\end{array}$ \\
\hline Safe and Effective Care Environment & $26 \%-38 \%$ \\
Coordinated Care & $17 \%-23 \%$ \\
Safety and Infection Control & $9 \%-15 \%$ \\
Health Promotion and Maintenance & $6 \%-12 \%$ \\
Psychosocial Integrity & $6 \%-12 \%$ \\
Physiological Integrity & $38 \%-62 \%$ \\
Basic Care and Comfort & $6 \%-12 \%$ \\
Pharmacological Therapies & $12 \%-18 \%$ \\
Reduction of Risk Potential & $9 \%-15 \%$ \\
Physiological Adaptation & $11 \%-17 \%$ \\
\hline
\end{tabular}

Items are selected and administered in this way until the computer makes a pass or fail decision. To complete this exam, candidates are allotted a total of six hours and are required to answer between a minimum of 75 items and a maximum of 265 items. ${ }^{[13-15]}$

Although there are benefits to this form of testing — such as ability to identify a candidate's clinical competence and ability to perform safely, increased test security, flexibility in test management, and immediate availability of scores ${ }^{[16]}$ — this form of testing is challenging not only for IENs but also for other Canadian students. Given their lack of familiarity with test content, structure, and mode of delivery, Canadian students need to familiarize themselves with different areas associated with the NCLEX-RN; for example, they must memorize lab values and therapeutic drug dosages for commonly used drugs. ${ }^{[17]}$ Further, according to the CNSA, ${ }^{[17]}$ students should be psychologically prepared for writing a computer adaptive exam. Notably, students should be aware that questions are presented, one at a time, on a computer screen, with no opportunity to go back and change an answer or to answer a question at a later time.

The transition from the old to the new nursing registration exam impacts educators as well as students; schools with limited available resources must try to provide students and faculty with access to relevant technology, while faculty bears the responsibility of preparing students to both learn and respond to questions in this mode of test delivery. To meet expectations of the NCLEX-RN, it is critical that educators embed computerized adaptive testing and associated forms of test taking in the nursing curriculum.

\subsection{Application of experiential education approaches in nursing education}

Content of the NCLEX-RN is based on and measured using the cognitive domain adopted from Bloom's taxonomy. The taxonomy outlines three domains of learning: cognitive, affective, and psychomotor. Further, it promotes higher forms of learning, such as analyzing and evaluating concepts and processes, over other forms, such as memorizing.

Nursing practice requires the application of higher order cognitive skills and abilities and complex thought processes, and most questions on the NCLEX-RN are written to align with this notion. Educators, therefore, are required to adequately prepare students to use problem-solving and criticalthinking skills in order to address the hierarchical steps in the cognitive domain. These are categorized from simple to complex and include content that addresses knowledge, comprehension, application, analysis, synthesis, and evaluation. ${ }^{[18,19]}$ This poses yet another challenge, as the application of Bloom's taxonomy now needs to be both addressed and integrated into nursing programs to better prepare candidates for this exam.

Faculty plays a vital role in aiding students to prepare for and pass the NCLEX-RN. Critical thinking is considered a priority in nursing education, so it is imperative both to begin addressing, assessing, and evaluating students' knowledge at an early stage and to apply techniques to enhance comprehension, application, analysis, and synthesis over the course of program. The low first-time pass rates of nurses educated outside the $\mathrm{US}^{[3,20]}$ underscore the importance of exploring strategies for increasing pass rates among IENs in Canada.

Research indicates that the present generation of students exhibits a preference for digital literacy, experiential learning, interactivity, and immediacy; increasingly, technology is being integrated into university courses to aid in student involvement and knowledge attainment. ${ }^{[21]}$ Current research studies that examine the application of experiential-education approaches in the context of teaching learning of novice health-care practitioners and IENs identify the benefits of technology in enhancing critical thinking, clinical decision making and reasoning, and clinical judgment. ${ }^{[12,22-25]}$ Given that the NCLEX-RN examination is administered using CAT, the application and integration of technology is imperative in nursing education. One form of technology that aligns with the format of NCLEX-RN questions is the use of clickers.

\subsection{Application of clickers in this study}

In this study, clickers were implemented as a form of experiential-education methodology to both introduce participants to how questions and items are presented on the NCLEX-RN and to provide them with a better understanding of question categories and content distribution. After being presented with questions based on the material covered in case scenarios, participants entered their responses using 
clickers. Once answers were entered, the aggregate results were immediately displayed in a table format, which provided immediate feedback and allowed for further discussion of rationales for correct answers.

According to Benner, Tanner \& Chesla, ${ }^{[26]}$ debating and reasoning through actual clinical-case scenarios facilitate students' acquisition of predictive clinical reasoning and clinical judgment. Given that nurses must constantly assess information and respond with sound judgment, efficiency, and care, the critical-thinking skills necessary for clinical judgment need to be developed. Using the results generated by clickers can aid in this development as they afford students an opportunity to debate their rationales concerning the scenarios and, by extension, to develop clinical reasoning and judgment.

The application of clickers was incorporated in this study as a methodology to enhance student interaction and learning and to provide opportunities for small-group problem solving. Students were presented with clinical situations and given limited information, from which they were to discern the most appropriate course of action for safe and effective nursing care.

\section{Methods}

The research question that guided this study is, will the application of experiential education approaches enhance NCLEX-RN scores of IENs?

\subsection{Participant demographics}

This study employed a purposive sample of ten participants who were enrolled in the IEN program at a university in Ontario. This IEN program is a 20-month, six-term program. The participants, who were all in the same cohort and in their first term while engaged in the study, were all women. None of the participants had practiced nursing in Ontario, but all had practiced outside Canada.

\subsection{Research design}

After consent was obtained, participants engaged in a series of six clinical-case scenarios that focused on nursing care of patients with different physiological complexities related to the respiratory, cardiovascular, peripheral vascular, abdominal, neurologic, and endocrine systems. These scenarios were scheduled over a six-week period. Before beginning the series of scenarios, and again after completing the series, participants wrote a mock NCLEX-RN. Due to budget constraints, CAT was not available to administer the test. However, while the delivery system differed, the questions on the pre and post exam mirrored the NCLEX-RN in both format and content.

Sixty questions were developed for clicker polling sessions 104 (ten for each of the six clinical case scenarios) and fifty for the mock exam; these questions aligned with the Bloom's taxonomy of higher order thinking and hierarchical steps in the cognitive domain. The questions were developed by the researcher and were based on 2013 NCLEX-RN Test Plan client needs categories and content distribution. They were formatted to align with NCLEX-RN question styles and, so, included multiple choice, fill in the blank, hotspot, and calculation questions; further the questions incorporated video and audio clips, graphs, charts, and tables. The associated clinical situations provided the participants with opportunities for the application of complex and critical thinking and decision-making. Clickers were used to answer ten questions associated with the content proceeded each clinical case scenario.

The use of clickers allowed an opportunity for knowledge, application, and analysis of content presented through the clinical situations, as participants determined the best or most appropriate, safe, and effective approach or action. The findings illustrate that the application of clickers allowed for immediate feedback, which afforded participants the opportunity for discussion and, thus, facilitated knowledge.

\section{RESULTS}

The participants' scores on the pre and post mock exams were collected and analyzed using paired sample $t$ test. A change in mean scores, as calculated using the paired sample $t$ test, would indicate whether participants' knowledge or scores increased or decreased for the whole group, though statistical significance cannot be ascertained without using statistical comparison methods. (The results of the paired sample $t$ test are illustrated Table 2). There was not a significant difference in scores from pretest $(\mathrm{M}=56.40, \mathrm{SD}=3.75)$ to posttest $(\mathrm{M}$ $=59.20, \mathrm{SD}=3.68$; see Table 3 ). Changes in the mean scores identify that performance increased slightly for the whole group. The results also indicate that the application of the intervention in this study slightly improved scores, though scores remained low. This could indicate that the participants did not know or understand the content administered or were not familiar with the format of the questions.

The confidence interval equals -2.80 with a $95 \%$ confidence interval of this difference ranging from -5.06 to -0.54 . Intermediate values used in calculation include $\mathrm{df}=9, \mathrm{SE}(\mathrm{d})=$ 0.998 .

The low scores on the post mock NCLEX-RN demonstrate that IENs are prepared for neither the content nor the question format of the NCLEX-RN, which raises concerns regarding the exam's impact on educators and nursing programs at large. 
Table 2. Results of the Paired Sample $t$ Test

\begin{tabular}{llll}
\hline Student & Pre score & Post score & Difference \\
\hline 1 & 54 & 60 & +4 \\
2 & 60 & 60 & 0 \\
3 & 52 & 50 & -2 \\
4 & 50 & 56 & +6 \\
5 & 56 & 60 & +4 \\
6 & 54 & 62 & +8 \\
7 & 60 & 62 & +2 \\
8 & 58 & 60 & +2 \\
9 & 60 & 60 & 0 \\
10 & 60 & 62 & +2 \\
Mean & 56.40 & 59.20 & 2.6 \\
\hline
\end{tabular}

Table 3. Pretest and Posttest Differences in Mean Scores and SD and SE(d)

\begin{tabular}{lll}
\hline Value & Pre score & Post score \\
\hline Mean & 56.4 & 59.2 \\
SD & 3.75 & 3.68 \\
SEM & 1.19 & 1.16 \\
\hline
\end{tabular}

Note. $t$ (2.8062), a two-tailed $p$ value equals .0205

\section{Limitations}

A number of limitations to this study have been identified. These include (a) sample size and composition, (b) length of study, (c) role of the researcher and position in relation to the study, and (d) setting where the research took place.

Although the sample size was small, it was appropriate for preliminary research and appropriate in the context of this study. ${ }^{[27]}$ The small number of students participating in this study, and their enrolment in the IEN program, limit the researcher's ability to generalize the findings to other populations. Given the size of the sample, the findings do not necessarily demonstrate represent IENs across all programs and cannot be generalized for this intervention. It should also be noted that research was conducted with the sample performing as a group, as opposed to individually, which could challenge the validity of the findings. Although the findings contribute to the literature on the challenges associated with the introduction of the new Canadian NCLEX-RN, a larger sample size may have generated more data. In future studies with a similar focus, a larger sample size may lead to diverse results and more extensive findings.

Another limitation is that the sample was not broken down by gender or culture, nor was it large enough to do so; nonetheless, these differences may have influenced the findings. This indicates the need to research nursing students by gender and culture, as these factors may influence one's culture of care and nursing practice.
Further, it should be noted that the research was conducted over a period of six weeks. The modest gains reported between the pretest and posttest scores may have been greater if the study had run over a course of an entire semester.

My role within the study, both as an educator in the program and as an "insider" may have influenced the findings. Although I was not teaching the participants at the time of the study, they knew me as an educator in the program. Participants' perception of me may have influenced their responses and actions during the discussion associated with the clinical-case scenarios and their engagement during the clicker-assisted question periods.

A further limitation is that the study was conducted at a single university with participants who were all in the same cohort of IENs and in the same stage (their 1st semester) of the program. The IENs were selected from an academic university-level program, rather than from other bridging programs available in Canada. The knowledge the participants had already gained through their enrolment in the program may have influenced both the thinking that occurred during data collection and the findings.

That the participants wrote the pre and post NCLEX-RN style mock exams on paper is another limitation-one that echoes the limited resources available to prepare students for this exam. The inability to administer this exam in its actual format is a challenge faced not only for this study but also in reality in other nursing programs. Participants were unable to take the exams in a real computerized environment, which prevented them from engaging in the adaptive testing method. Future studies can explore similar methodology using CAT.

\section{Discussion}

The findings of this study indicate an urgent need for application and integration of content and the use of experientialeducation approaches to enhance NCLEX-RN scores among Canadian students. This has great implications for student and faculty alike. We must explore approaches that aid students in developing confidence with and meeting the expectations of the NCLEX-RN; thus it is imperative to provide them with opportunities to develop critical-thinking skills and to explore and engage with CAT. The lack of significant change to the pre and post mock-exam scores indicates a need to expand the research to examine the ability of other experiential-education approaches to engage both students and faculty. It is important to consider approaches that improve students' level of understanding by raising the level of participation, fostering communication, and clarifying misunderstandings, and by embedding assessment as a learning activity rather than following the summative assessment 
approach.

In the study, the experiential-education approaches used included the application of clickers and clinical scenarios. The real-life clinical scenarios provided students with an opportunity to practice and learn critical thinking and decisionmaking skills and to apply higher order cognitive skills; the question sessions bridged the gap between classroom learning and clinical practice, which is particularly necessary for novice nurses with limited clinical background. The application of clinical-case scenarios followed by audience response system (ARS) technology allowed students not only to see if they answered correctly, but also to compare their responses to those of the other members of the class. Moreover, the information was then used to clarify lecture material and stimulate class discussion or other types of collaborative learning. ${ }^{[28]}$

Further examples of experiential-education methodologies include unfolding clinical-case studies, flipped-classroom design, and simulation-based pedagogy that incorporates the application of high technology role-play and standardized patient methods. Incorporating the use of these methods will (a) enhance student engagement and reflection on how to take the best course of action in a clinical setting, (b) provide a safe way to learn how to make high-risk clinical decisions, and (c) provide a bridge between classroom learning and clinical practice, which is particularly necessary for IENs.

Given that instructors play a pivotal role in students' success rates, it is important for faculty to familiarize themselves with the style of the NCLEX-RN and to be aware of how it differs from the CRNE. One notable difference is the distribution of content of testable material, as $62 \%$ of the NCLEX-RN is based on physiological integrity. ${ }^{[13]}$ As both the content and format of the NCLEX-RN differ from that of the CRNE, the manner in which faculty has taught and delivered content in the past is an area that requires close attention. It is imperative to first educate the educator on how these challenges can best be addressed in the classroom in order to foster student success.

To better prepare students for the NCLEX-RN, educators should familiarize themselves with the NCLEX-RN format, content distribution, and delivery method. Educators might also familiarize themselves with the NCSBN's practice analysis report, which provides an understanding of how questions are created and weighted to reflect the importance of tasks and skills and their application in the clinical milieu. The results of the practice analysis are aligned with the test plan, giving educators information to prepare entry-level nurses. ${ }^{[4]}$

This study answers a number of questions about the impli- cations and impact the introduction of the NCLEX-RN will have for IENs and Canadian nursing programs. Nonetheless, many questions remain: How does preparing for this exam meet expectations for the Canadian clinical and healthcare arena, the overall nursing landscape, and associated patient-care outcomes? How can we ensure issues associated with Inuit/First Nations/northern populations will be incorporated? How can we ensure that Canadian candidates will not be expected to answer questions on skills that are not considered entry-level in parts of Canada (i.e., the use ventilators and arterial lines, the interpretation of ECGs, etc.)? How can we align the entry-to-practice (ETP) competencies in Canada with the items that will be on the exam?

Other researchers from different backgrounds - such as practice leaders, academics, and policy makers - may better address these remaining questions, as they may bring different perspectives to the changes inherent to the administration of the exam to a new generation of nurses and its integration in the transition to practice.

\section{A Summary of further recommendations for research}

Further research is needed to augment the findings from this research. Other scholars might consider the following research activities:

(1) Explore how results of the NCLEX-RN influence patient-care outcomes.

(2) Investigate the application of other experientialeducation approaches; in particular, conduct research within other courses in the nursing curriculum.

(3) Explore the challenges associated with the implementation of CAT into Canadian nursing programs.

(4) Compare first-time pass rates of Canadian candidates to those of US candidates.

(5) Conduct more pre and post studies to further explore the application of CAT in the Canadian context.

(6) Research how critical thinking, developed through the use of other experiential-education methods such as flipped-classroom and simulation-based approaches, impacts NCLEX-RN scores.

(7) Explore the NCLEX-RN scores of novice nurses from different geographical and educational backgrounds.

(8) Integrate the PearsonVUE Alternative Item Format Online Tutorial (which gives students the opportunity to practice completing alternative item format questions) into classroom practice and explore students' pre and post scores on mock NCLEX-RN exams.

\section{Conclusion}

Through the course of the research study, it became clear that there is a need to explore more ways to prepare IENs 
and other nursing students for success on the NCLEX-RN. The need to study how best to assist faculty to develop new resources that align with the NCLEX-RN and to embed them in the curriculum also became apparent.

It is important to support IENs in their transition to practice in Ontario and to ensure that they are equipped to practice safely. The process of integration of and transition to a new exam can be overwhelming, given the unfamiliar format and administration of the exam, not to mention the often significant differences between Canadian and US health-care systems and specialized nursing language. These factors make the process of integration and adjustment difficult.

The findings suggest a need for further discussion in the academic literature regarding how to better prepare IENs and other nursing students. That being said, it is important to guide and facilitate education through new and innovative ways that illustrate the complexity of nursing care and consider different cultures of care. Acknowledging and recognizing these will both enhance clinical decision making and reasoning and critical thinking and support novices transition to practice.

This study has advanced the understanding of both the challenges associated with the NCLEX-RN within the Canadian context and the ways that may support or enhance success rates among IENs and other Canadian nursing students.
Knowledge gained in this study represents merely the tip of the iceberg in understanding how IENs learn. Although this study provided some insight about one form of experientialeducation approach, there is a need to explore more methods of teaching and learning pertaining to content delivery and application. Further research-including similar studies that incorporate samples that are larger or that include participants from a wider range of undergraduate nursing programs-is needed in this area.

The ideas raised in this article will better equip faculty members to implement pedagogical interventions and adjust curriculum based on pedagogical implications of new research and develop educational resources for IENs and other cohorts of nursing students through the course of their program. Further, these ideas represent a means of facilitating the development of support resources and the transition to practice in Ontario. The findings provide insight into the implications and challenges of the NCLEX-RN for nursing students and faculty alike and, thus, contribute to the overall development of nursing knowledge and pedagogy.

\section{ACKNOWLEDGEMENTS}

The YUFA Teaching Leaning Development Grant

\section{CONFLICTS OF INTEREST Disclosure}

The author declares that there is no competing interests.

\section{REFERENCES}

[1] Canadian Council of Registered Nurse Regulators. NCLEX frequently asked questions for Canadian educators and students: NCSBN examinations. 2013. Available from: http: //www. ccrnr. ca/index_files/CCRNRNCLEXEducation.html

[2] Canadian Council of Registered Nurse Regulators. NCLEX frequently asked questions for Canadian educators and students: NCSBN examinations. 2013. Available from: http: //www . ccrnr. $\mathrm{ca} /$ index_files/CCRNRNCLEXEducation.html

[3] National Council of State Boards of Nursing. NCLEX statistics from NCSBN. 2014a. Available from: https ://www.ncsbn.org/Tabl e_of_Pass_Rates_2014.pdf

[4] National Council of State Boards of Nursing. Computer adaptive testing FAQs. 2015a. Available from: https : //www.ncsbn.org/ 4700.htm

[5] National Council of State Boards of Nursing. NCLEX Statistics from NCSBN. 2015b. Available from: https ://www.ncsbn.org/Tabl e_of_Pass_Rates_2015_(3).pdf

[6] McGuire M, Murphy S. The internationally educated nurse: Wellresearched and sustainable programs are needed to introduce internationally educated nurses to the culture of nursing practice in Canada. Canadian Nurse. 2005; 101(1): 25-29. PMid:15776715.

[7] International Migration Research Centre. (2014). Immigration policy changes and entry to practice routes for internationally educated nurses (IENs). Available from:
http://imrc.ca/wp-content/uploads/2013/09/SSHRC

-FINAL-mar-14-2014typeset3_1.pdf

[8] Home G. Canada's policy framework for the utilization of internationally educated nurses. Queen's Policy Review. 2011; 2(2): 37-53.

[9] Touchstone Institute. Internationally Educated Nurses Competency Assessment Program (IENCAP). 2015. http: //www . touchstone institute.ca/competency-assessment/iencap.aspx

[10] College of Nurses of Ontario. The national competencies of entry level registered nurse practice. 2014b. Available from: http://www.cno.org/Global/docs/reg/41037_Entr yToPracitic_final.pdf

[11] Peisachovich E. Exploring the experience and understanding of clinical judgment of IENs transitioning to nursing practice in Ontario. Faculty of Education, York University. (Unpublished doctoral dissertation). 2014

[12] Peisachovich EH. The experience and understanding of clinical judgment of internationally educated nurses. Journal of Nursing Education and Practice. 2015; 5(8): 33-43. http://dx.doi.org/10.54 30/jnep.v5n8p33

[13] National Council of State Boards of Nursing. NCLEXRN test plan. 2013. Available from https ://www.ncsbn.org/2013_NCLEX_R N_Test_Plan.pdf

[14] National Council of State Boards of Nursing. 2014b. 2014 NCLEX Candidate examination bulletin. Available from: https ://www.nc sbn.org/2014_NCLEX_Candidate_Bulletin.pdf 
[15] Lavin J, Rosario-Sim MG. Understanding the NCLEX: How to increase success on the revised 2013 examination. Nursing Education Perspectives. 2013; 34: 196-198. http://dx.doi.org/10.5480 /1536-5026-34.3.196

[16] Georgiadou E, Triantafillou E, Economides AA. Evaluation parameters for computer-adaptive testing. British Journal of Educational Technology. 2006; 37: 261-278. http://dx.doi.org/10.1111/j $.1467-8535.2005 .00525 . \mathrm{x}$

[17] Canadian Nursing Student Association. (n.d.). Tips for nursing students. Available from: http://www.cnsa.ca/files/files/NC LEX_Tips $\% 20$ for $\% 20$ Students $\% 20-\% 20$ FINAL .pdf

[18] Anderson LW, Krathwohl, D. (Eds.). A taxonomy for learning, teaching, and assessing: A revision of Bloom's taxonomy of educational objectives. New York, NY: Longman. 2001.

[19] Bloom BS, Engelhart MD, Furst EJ, et al. Taxonomy of educational objectives: The classification of educational goals. Handbook I. Cognitive domain. New York, NY: David McKay. 1956.

[20] National Council of State Boards of Nursing. NCLEX statistics from NCSBN. 2013b. Available from: https://www.ncsbn.org/Tabl e_of_Pass_Rates_2013.pdf

[21] Oblinger DG, Oblinger JL. Educating the net generation. 2005. Available from: https://net.educause.edu/ir/library/pdf/pu b7101.pdf

[22] Clark D. Bloom's taxonomy of learning domains: The three types of learning. Big Dog \& Little Dog's Performance Juxtaposition. Ed- monds, WA: Author. 2010. Available from: http://www.nwlink .com/ donclark/hrd/bloom.html

[23] Forehand M. Bloom's taxonomy. In M. Orey (Ed.), Emerging perspectives on learning, teaching, and technology. 2005. Available from: http://epltt.coe.uga.edu/index.php?title= Bloom\%27s_Taxonomy

[24] Jeffries PR. (Ed.). Simulation in nursing education from conceptualization to evaluation (2nd ed.). New York, NY: National League for Nursing. 2012.

[25] Peisachovich EH. The importance of intercultural fluency in developing clinical judgment. Journal of Nursing and Health Care. 2015b; 2(2): 53-59.

[26] Benner P, Tanner C, Chesla C. Expertise in nursing: Caring, clinical judgment, and ethics. (2nd ed.). New York, NY: Springer Publishing. 2009.

[27] Wagner D, Bear M, Sander J. (Turning simulation into reality: increasing student competence and confidence. Journal of Nursing Education. 2009; 48: 465-467. Available from: http://www .healio.com/nursing/journals/jne/2009 -8-48-8/\%7Bb0d809f7-826f-4f c5-ad9f-fa4913ea $72 \mathrm{a} 0 \%$ 7D/turning-simulation-into-reality-increasing-s tudent-competence-and-confidence. PMid:19681537. http://dx.doi.org/10.3928/01484834-20090518-07

[28] Center for Excellence in Learning and Teaching (CELT). A model of learning objectives. Iowa State University. 2011. Available from: http://www.celt.iastate.edu/teaching/RevisedB looms1.html 